\title{
A Simple Nonlinear Dynamic Model for Unemployment: Explaining the Spanish Case
}

\author{
João Ricardo Faria ${ }^{1}$ and Miguel A. León-Ledesma ${ }^{2}$ \\ ${ }^{1}$ IPED, University of Texas at El Paso, TX 79968, USA \\ ${ }^{2}$ Department of Economics, Keynes College, University of Kent, Canterbury CT2 7NP, UK
}

Correspondence should be addressed to João Ricardo Faria, rfaria2@utep.edu

Received 22 January 2008; Accepted 14 June 2008

Recommended by E. Casetti

Spanish unemployment is characterized by three distinct regimes of low, medium, and high unemployment and by a fast transition between them. This paper presents a simple nonlinear dynamic model that is able to explain this behavior with multiple equilibria and jumps describing the transition between equilibria. The model has only a small number of parameters capturing the fundamentals of labor markets and macroeconomic and institutional factors. The model is capable of generating unemployment dynamics that encompass the "unique" natural rate hypothesis, the structuralist hypothesis, and the hysteresis hypothesis.

Copyright (C) 2008 J. R. Faria and M. A. Leon-Ledesma. This is an open access article distributed under the Creative Commons Attribution License, which permits unrestricted use, distribution, and reproduction in any medium, provided the original work is properly cited.

\section{Introduction}

Spain's unemployment performance has been the focus of much theoretical and empirical literature. (On Spanish unemployment see, among others, Bentolila and Blanchard [1], Bentolila and Dolado [2], Dolado and Jimeno [3], Dolado et al. [4], Juselius and Ordonez [5], Romero-Ávila and Usabiaga [6].) After a period of very low unemployment, during the 1980s and 1990s, Spain's unemployment rose to the highest of the OECD group reaching levels above $20 \%$. In recent years, however, Spain has become the main source of employment creation in Europe, which has led to a dramatic fall in the unemployment rate to levels of around $8 \%$. What is remarkable about Spanish unemployment during this period is that it appears to have jumped very quickly from a low to a high unemployment equilibrium. As a result, any model of the Spanish unemployment should be able to yield multiple equilibria and a fast transition between them.

This paper puts forward a simple nonlinear dynamic model that is able to generate these regime changes and their rapid transition with a small number of parameters capturing 


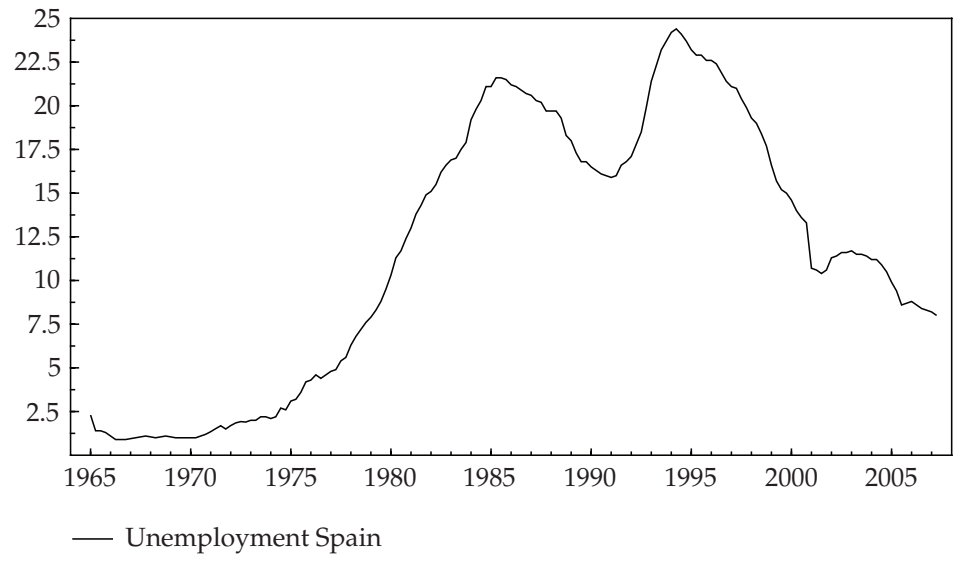

Figure 1: Spanish unemployment 1965:1-2007:2.

the main factors of Spanish unemployment, including labor market fundamentals and macroeconomic and institutional factors that may have acted upon it. In the model, changes in the institutional characteristics of the labor market or macroeconomic conditions can potentially lead to large unemployment equilibrium jumps. The model is also able to encompass the three main unemployment dynamics theories in the literature, namely, the natural rate hypothesis, the structuralist hypothesis, and unemployment hysteresis.

After briefly characterizing the Spanish unemployment in Section 2, we present the model in Section 3. Section 4 provides some links between the empirical characterization and the theoretical model, while Section 5 concludes.

\section{Characterizing spanish unemployment}

As mentioned earlier, the evolution of Spanish unemployment has been characterized by three main phases (see Figure 1). The first one is between 1965 and the second half of the 1970s, where unemployment is very low at levels averaging about $2 \%$ up to 1977 . In the second phase, between 1980 and the second half of the 1990s, unemployment increases dramatically and stays at a very high level for almost 30 years. Indeed, unemployment rates reach a peak in 1994 at $24 \%$. The third phase is characterized by a rapid decrease in unemployment between the second half of the 1990s and 2007. The current unemployment rate in 2007 has recovered to levels of about $8 \%$ (all the data is quarterly and refers to OECD's standardized unemployment rate).

These rapid changes in unemployment rates have coincided with important events and reforms taking place in the Spanish economy in the last 30 years. (For an overview of labor market reforms in Spain see Ferreiro and Serrano [7].) The low Spanish unemployment during the 1960s and early 1970s reflected a rapidly growing economy during the catch-up process, but also the fact that migration absorbed large part of the excess labor force. With the process of democratic transition, the government embarked on important reforms of the labor market and taxation systems to mimic the European postwar welfare state institutions. Important events were the approval of the Workers' Statute (1977) and the tax reform (1977-78). The first legally recognized trade unions and collective bargaining and gave preference to permanent 
contracts, whereas the second modernized the tax system with the introduction of personal income tax and corporate tax to substitute the archaic tax system of the Francoist era. The new system also extended unemployment benefits to job losers. These changes coincided with a deep economic recession, deindustrialization, and high inflation. The high unemployment rates that ensued planted the seeds for another labor market reform in 1984 to introduce more flexibility especially for temporary employment. (This period also saw several tax reforms and the introduction of the VAT as general indirect tax in preparation for the Spanish entry in the European Community.) This reform also coincided with an economic recovery which saw unemployment rates falling but only to levels close to 16\%. The ERM crisis in 1991 generated another deep recession and unemployment rates increased dramatically in the following three years. This led to another labor market reform in 1994 to give flexibility to the wage setting process with preference for decentralized bargaining. Another two reforms were introduced in 1997 and 2002 to increase the flexibility of the labor market and reduce the duality generated by the increase in temporary work. The reforms during the 1990s and 2000s also changed (reduced) substantially unemployment benefits and eligibility criteria. These changes also coincided with tax reforms to improve the efficiency of tax collection, reduce the number of tax brackets, and, during the 2000s, reduce the high marginal tax rates. All these reforms took place in a context of strong trade liberalization, especially with the accession to the EU (then European Economic Community) in 1986, deregulation of goods markets and privatization.

Against this background, the rapid and significant unemployment changes can be characterized as a multiple equilibria pattern. To illustrate this point, we can use a Markov switching in mean (MSM) model as put forward by Hamilton [8], to characterize the dynamics of the Spanish unemployment rate. We estimated the following MSM dynamic model for the unemployment rate:

$$
\left[u_{t}-\mu\left(s_{t}\right)\right]=\sum_{p=1}^{P} \phi_{p}\left[u_{t-p}-\mu\left(s_{t}\right)\right]+\varepsilon_{t}
$$

where $u_{t}$ is the unemployment rate, $p$ is the lag augmentation, $\phi_{p}$ are autoregressive coefficients, $\varepsilon_{t}$ is an i.i.d. $N(0, \sigma)$ error term, and $\mu\left(s_{t}\right)$ is the mean of the unemployment rate that depends on $s_{t}$ which is the unobservable realization of $M$ states that is governed by a discrete-time, discrete-state Markov stochastic process. This process is defined by the transition probabilities $\operatorname{Pr}\left(s_{t+1}=j \mid s_{t}=i\right)=p_{i j}$ with $\sum_{j=1}^{M} p_{i j}=1, \forall i$.

This is a simple representation of a multiple equilibria process for unemployment (see León-Ledesma and McAdam [9] for the case of Eastern European countries). In our case, we allow a maximum number of 3 states $(M=3)$, that is, the variable is allowed to switch between three different regimes $s_{t}=1,2,3$. (The model allows for a lag augmentation of 4.)

Estimation of the model using the EM algorithm yields three clear states of high, medium, and low unemployment. The results in Table 1 show the mean unemployment rates in regimes 1,2, and 3, the transition probabilities between regimes, and the timing of the three regimes. The actual and mean unemployment, together with its estimated mean and the smoothed transition probabilities are plotted in Figure 2. The results show a dramatic increase in unemployment in 1978 from its previous $2.2 \%$ mean. For a short period it moves into the second regime with a mean of $10.8 \%$ and then rapidly moves in 1982 into the third highunemployment regime (mean 19.5\%). By 1999, unemployment exits the high-unemployment regime into the medium-unemployment one. The three regimes are very persistent, especially 
Table 1: Markov-switching model for Spain's unemployment.

\begin{tabular}{|c|c|c|c|}
\hline & \multicolumn{3}{|c|}{ Regime means } \\
\hline & Mean & \multicolumn{2}{|c|}{ St. error } \\
\hline Regime 1 & 2.216 & \multicolumn{2}{|c|}{0.316} \\
\hline Regime 2 & 10.77 & \multicolumn{2}{|c|}{0.407} \\
\hline \multirow[t]{3}{*}{ Regime 3} & 19.52 & \multicolumn{2}{|c|}{0.294} \\
\hline & \multicolumn{3}{|c|}{ Transition probabilities } \\
\hline & Regime 1 & Regime 2 & Regime 3 \\
\hline Regime 1 & 0.9815 & 0.01853 & $1.27 \mathrm{E}-28$ \\
\hline Regime 2 & 3.09E-12 & 0.978 & 0.022 \\
\hline \multirow[t]{3}{*}{ Regime 3} & 2.61E-15 & 0.0142 & 0.9858 \\
\hline & \multicolumn{3}{|c|}{ Regime duration } \\
\hline & $\%$ Observ. & \multicolumn{2}{|c|}{ Duration } \\
\hline Regime 1 & 53 & \multicolumn{2}{|c|}{53.96} \\
\hline Regime 2 & 46.5 & \multicolumn{2}{|c|}{45.45} \\
\hline \multirow[t]{2}{*}{ Regime 3} & 70.5 & \multicolumn{2}{|c|}{70.42} \\
\hline & \multicolumn{3}{|c|}{ Regime dating } \\
\hline Regime 1 & \multicolumn{3}{|c|}{ 1965:1-1978:1 } \\
\hline Regime 2 & \multicolumn{3}{|c|}{$1978: 2-1981: 4$} \\
\hline & \multicolumn{3}{|c|}{ 1999:4-2007:2 } \\
\hline Regime 3 & \multicolumn{3}{|c|}{ 1982:1-1999:3 } \\
\hline & \multicolumn{3}{|c|}{ AIC } \\
\hline$M=1$ (linear) & \multicolumn{3}{|c|}{6.92} \\
\hline$M=2$ & \multicolumn{3}{|c|}{5.57} \\
\hline$M=3$ & \multicolumn{3}{|c|}{4.65} \\
\hline
\end{tabular}

regimes 1 and 3 (low and high) as the probabilities of transition out of the current regime are very low. Following Psaradakis and Spagnolo [10], we also report the Akaike information criterion (AIC) to select the number of states which favors the choice of $M=3$.

The reported changes in equilibrium unemployment appear to coincide with some of the major labor market and tax reforms previously identified. They also coincide with major changes in economic performance, although these events may not be independent as some of the reforms took place after policy makers realized the need for reform and achieved sufficient political consensus. Hence, the Spanish unemployment can be characterized by multiple equilibria, where regime changes coincide with major economic and institutional changes in the Spanish economy. For this reason, a model of the Spanish unemployment dynamics has to be able to yield multiple equilibria as a consequence of internal and external changes in the labor market, and rapid transition between states.

\section{The model}

The model describes the time evolution of Spanish unemployment, $\dot{u} \equiv d u / d t$, as a result of two different forces, which we call internal and external. The internal forces are the labor market fundamentals, the ones that lie beneath the labor market equilibrium, affecting labor supply and labor demand. These internal forces include workers' and trade unions' preferences, outside options (such as unemployment benefits), bargaining power, firms' technology, and 


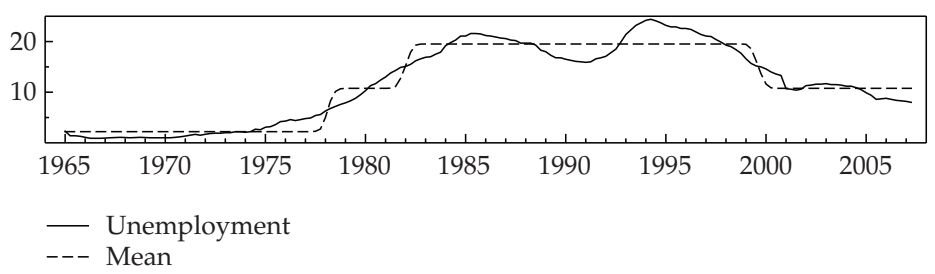

(a)

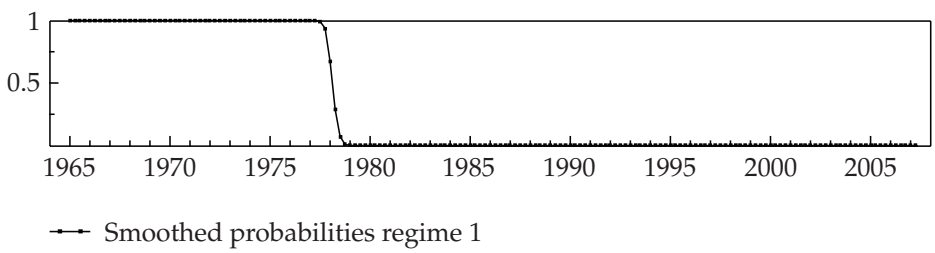

(b)

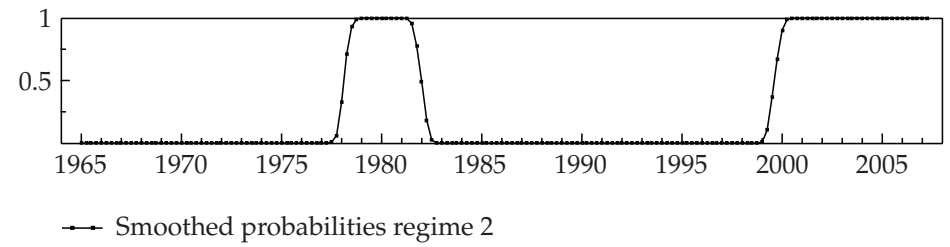

(c)

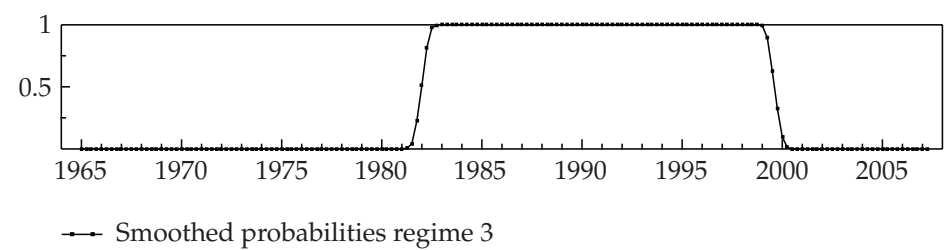

(d)

Figure 2: Unemployment and regime probabilities for Spain.

market power. These variables are all arguments of the internal forces function, denoted by $F(u, X)$, where $X$ is the vector of internal forces.

The external forces are external interventions aimed at reducing unemployment, affecting labor market equilibrium besides labor market fundamentals. Among these external forces are macroeconomic policies and institutional changes related to fiscal and monetary policies and goods markets. These forces are represented by the function $G(u, Y)$ where $Y$ is the vector of external forces.

Unemployment evolves according to

$$
\dot{u} \equiv F(u, X)-G(u, Y) \text {. }
$$

It is clear that in the steady state we have that $u=u(X, Y)$, which is the desired result of any model that aims at describing unemployment as a function of labor market fundamentals and institutional and macroeconomic characteristics of the economy.

However, this standard model is not a priori equipped to deal with multiple equilibria or to explain the transitions from one equilibrium unemployment to another. If Spanish 
unemployment is characterized by several distinct regimes, it has multiple equilibria. Moreover, the change from one regime to another appears to have been very fast. As a consequence, a reasonable model of Spanish unemployment has to generate at least two steady state equilibria and be able to explain the factors that led to a fast transition between them. The model in (3.1) can do this by assuming very simple nonlinear dynamics.

Starting with function $F(u, X)$, and in order to keep the model as simple as possible, it is enough to assume that internal forces act to limit growth towards an upper limit, that is, unemployment cannot affect the whole working population, normalized to one. Therefore, $F(u, X)=r(X)(1-u)$, where the relative rate of change $r$ is a function of $X$.

In the same vein, let us assume that function $G(u, Y)$ captures all external interventions aimed at reducing unemployment. For instance, it is well-known that government's taxes may affect unemployment (e.g., [11-14]), so the government may be tempted to put forward tax schemes to reduce unemployment; the same holds true for other macroeconomic policies and institutional changes (e.g., [15-17]). The main characteristic of our function $G(u, Y)$ is that it has an upper limit to the rate of unemployment reduction, that is, when unemployment is too high, these external forces may not be effective in reducing unemployment due to the limit imposed by labor market tightness. This means that there is an upper limit to the rate of reduction of unemployment due to these external interventions in the labor market. This is a reasonable assumption because all these forces are external to the fundamentals of the labor market. A simple formulation of function $G(u, Y)$ is the following: $G(u, Y)=b(Y)\left(u /\left(a^{2}+u^{2}\right)\right)$, where the upper limit $b$ is a function of $Y$, and $a$ is a positive constant.

Substituting $F(u, X)$ and $G(u, Y)$ into (3.1) yields

$$
\dot{u}=r(X)(1-u)-b(Y) \frac{u}{a^{2}+u^{2}} .
$$

This model has multiple equilibria, the equilibrium values of unemployment $u$ must satisfy

$$
\dot{u}=0 \Longrightarrow r(X)(1-u)=b(Y) \frac{u}{a^{2}+u^{2}} .
$$

In order to make the analysis of the model simpler, we scale the equation by making $\lambda=u / a$, and multiplying by $a / b(Y)$, yielding

$$
\frac{a}{b(Y)} r(X)(1-a \lambda)=\frac{\lambda}{1+\lambda^{2}} .
$$

The points where the left and right-hand sides of (3.4) intersect are the equilibria for $\lambda$, and, equivalently, $u$. The two sides of (3.4) are plotted in Figure 3. The left-hand side is a straight line with intercepts $a(r(X) / b(Y))$, and $1 / a$. This last intercept is by definition fixed, while the first one varies with the parameters of $r(X)$ and $b(Y)$. The right-hand side of (3.2) is a peaked curve, which describes the external effort in reducing unemployment. This curve crosses the origin and is asymptotic to the $\lambda$ axis at high unemployment. The equilibria for unemployment are defined where the straight line intersects the peaked unemployment reducing curve. The number and location of these intersections depends on the parameters of $r(X)$ and $b(Y)$.

Figure 3 describes the case of two equilibria (low and high unemployment) and also shows that any given change in $r(X)$ and $b(Y)$, that increases the intercept $a(r(X) / b(Y))$, 


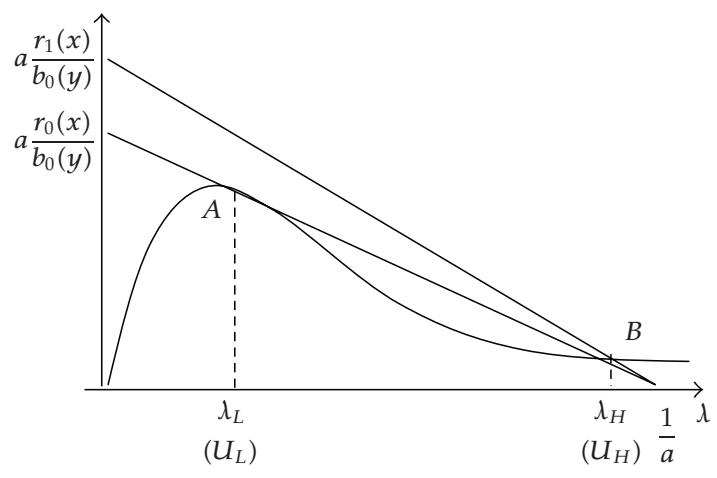

Figure 3: From low to high unemployment equilibrium.

may trigger a jump in the equilibrium rates of unemployment from low to high. As a consequence, this model is able to explain the evolution of the Spanish unemployment from a low equilibrium to a high equilibrium in such a short period of time given any small change in the parameters of $r(X)$ and $b(Y)$.

An example of the workings of the model is the following. The economy is initially at point $A$ and the government changes the eligibility rules of unemployment benefits increasing the pool of unemployed receiving benefits. This makes the function $r(X)$ increase, holding everything else constant, shifting the slope of the straight line to the right which triggers a major change from a low to high unemployment equilibrium as in point $B$. This is one of the hypotheses entertained by Blanchard and Jimeno [18] when explaining differences in unemployment performance in Spain and Portugal. As mentioned earlier, the democratic transition period was characterized by the passing of the workers statute and an extension of unemployment benefits to displaced workers in the late 1970s and early 1980s (see [19]). From the point of view of our model, this can trigger a rapid and dramatic increase in unemployment equilibrium.

The reverse is also true, the model is able to generate a jump from high unemployment to low unemployment, and this case is depicted in Figure 4. The economy is initially in a high unemployment equilibrium and for any slight change in the parameters of $r(X)$ and $b(Y)$, triggering a fall in $a(r(X) / b(Y))$, this leads to a jump from the high to a low unemployment equilibrium. As an example, the economy is initially at point $A$ and the government decreases tax progressivity. For countries characterized by industrial bargaining, such as Spain, Brunello and Sonedda [20] found that this government action may decrease unemployment. (See also Raurich et al. [21] for a model that explains the European unemployment hysteresis phenomenon in terms of fiscal policies.) Referring to Figure 4, a reduction in tax progressivity increases $b(Y)$, holding everything else constant, shifting the straight line to the left, making the economy jump from the high to low unemployment equilibrium, as in point $B$.

The model can be generalised to represent more complex internal forces, such as a logistic growth (which implies multiplying the function $F(\cdot)$ by $u$ and introducing an exogenous term representing the carrying capacity of the labour market). In this case, the dynamics remain the same as long as a quadratic term for $u$ appears on the numerator of the external forces function. An analogous case is analyzed by Ludwig et al. [22], who also discuss in depth the cusp catastrophe that characterizes this type of phenomena. A cusp catastrophe 


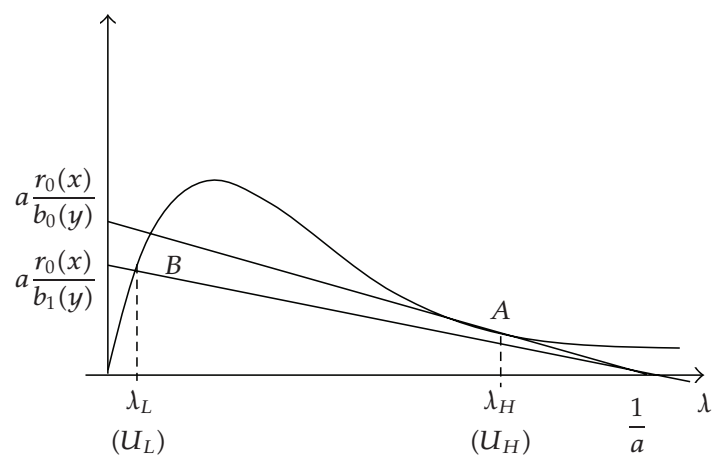

Figure 4: From high to low unemployment equilibrium.

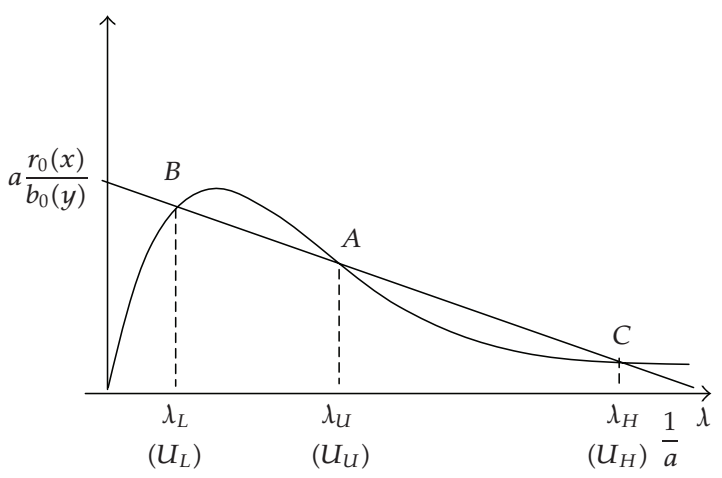

Figure 5: Unstable equilibrium.

describes the case of a quickly adjusting variable and two parameters. In the case of our model, this variable is unemployment and $r$ and $b$ are the parameters (the classic references of catastrophe theory are Thom [23] and Zeeman [24]).

These simple nonlinear dynamics can also encompass the case of three equilibria, one of them is an unstable equilibrium. Figure 5 represents this case. The economy is initially at point $A$, which is an unstable equilibrium. Any positive or negative shock can then move the economy towards the stable equilibria, which are the high or low unemployment equilibria (points $B$ and $C$, resp.). All that is necessary for this large unemployment change is a temporary shock. In this respect, the model can generate dynamics similar to those emphasised by the unemployment hysteresis literature (see Blanchard and Summers [25] and Røed [26]). This is because temporary shocks can have permanent effects on unemployment. This is an important feature of the model.

The empirical literature on unemployment dynamics has distinguished between three competing views, namely, the "unique" natural rate Friedman-Phelps hypothesis, the structuralist hypothesis represented by Phelps [15], and the hysteresis hypothesis (See, amongst many others, Camarero et al. [27]). Although hysteresis is usually represented as a unit root in unemployment, Jaeger and Parkinson [28] have shown that all that is necessary for hysteresis is that temporary shocks change the equilibrium unemployment. For the structuralist hypothesis, however, changes in equilibrium unemployment stem 


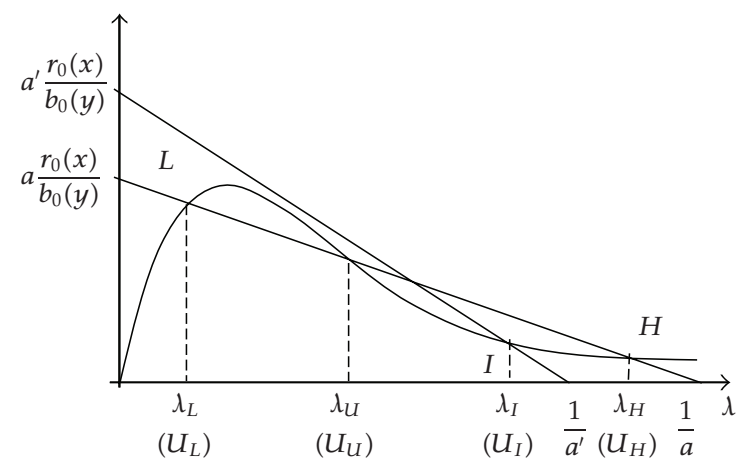

Figure 6: Three stable equilibria.

from permanent shocks, which in our model are generated by changes to the parameters governing external or internal labour market forces. Hence our model is capable of generating unemployment dynamics that encompass these two hypotheses (it is also possible, e.g., that a very large temporary shock changes the incentives that policy makers face, leading to a permanent change in, e.g., unemployment eligibility, the level of protection, and bargaining systems. This seems to be a relevant hypothesis for the Spanish case). The Friedman-Phelps natural rate case would simply arise in the particular case in which there are no changes in the parameters governing functions $r(X)$ and $b(Y)$.

\section{Reconciling empirical results with the model}

Our empirical results show that over the 1965-2007 time horizon, the Spanish unemployment jumped from regime 1 to regime 2 to regime 3, and then back to regime 2 (as in Figure 2). How can these three regimens be reconciled with the two stable equilibria of the topology envisaged by the formal model and by Figures 3, 4, and 5?

Evidence and theory appear to be at odds since the latter allows for either a single high stable equilibrium unemployment, or for a single low stable equilibrium unemployment, or for two stable (one high and one low) equilibria and one intermediate unstable equilibrium. In other words there are three regimens and at most two stable equilibria (we thank one of the referees and an associate editor for this observation). Here we try to reconcile the existence of three regimes for Spanish unemployment with our theoretical arguments.

In order to tackle this issue consider Figure 6, which reproduces Figure 5 and includes a new straight line in which we assume that parameter $a$ has changed exogenously to $a^{\prime}, a^{\prime}>a$, and the new straight line has intercepts $a^{\prime}(r(X) / b(Y))$, and $1 / a^{\prime}$. Notice that the intercept $1 / a^{\prime}$ is to the left of the intercept $1 / a$. The figure has now three stable equilibria, including one stable equilibrium for intermediary unemployment.

The reconciliation between our empirical results and the model runs along the following lines. The economy is initially at low unemployment equilibrium, L. There is an exogenous change in the parameter $a$ to $a^{\prime}$, which leads the economy to a new intermediary, and stable, unemployment equilibrium, I. In that case, any given change in $r(X)$ and $b(Y)$ 一that increases the intercept $a^{\prime}(r(X) / b(Y))$-may trigger a jump in the equilibrium rates of unemployment from intermediary to high, $\mathrm{H}$. The economy may stay at the high unemployment equilibrium 
for a while and new changes in $r(X)$ and $b(Y)$ that decrease the intercept $a^{\prime}(r(X) / b(Y))$ may trigger another jump in the equilibrium rates of unemployment from high to intermediary. Indeed, one can also think of these dynamics as a result of several changes in parameter $a$ alone as, for instance, a change from $a$ to $a^{\prime}$ and then back to $a$. This explanation shows that the formal model can also encompass a case with 3 stable equilibria as long as we allow for parameter $a$ to change exogenously.

\section{Concluding remarks}

The Spanish unemployment over the last 40 years is characterized by three regimes of low, medium, and high unemployment and by a fast transition between them. This paper puts forward a simple nonlinear dynamic model that is able to generate multiple unemployment equilibria and rapid transition from low to high unemployment. Additionally, the model is able to represent, with a small number of parameters, the main determinants of unemployment as represented by the fundamentals of labor market and macroeconomic and institutional factors. The model is capable of generating unemployment dynamics that encompass the "unique" natural rate hypothesis, the structuralist hypothesis, and the hysteresis hypothesis.

\section{Acknowledgment}

The authors would like to thank, without implicating, three anonymous referees and one associate editor for comments.

\section{References}

[1] S. Bentolila and O. J. Blanchard, “Spanish unemployment," Economic Policy, vol. 10, pp. 233-281, 1990.

[2] S. Bentolila and J. J. Dolado, "Labour flexibility and wages: lessons from Spain," Economic Policy, vol. 9, no. 18, pp. 53-99, 1994.

[3] J. J. Dolado and J. F. Jimeno, "The causes of Spanish unemployment: a structural VAR approach," European Economic Review, vol. 41, no. 7, pp. 1281-1307, 1997.

[4] J. J. Dolado, C. García-Serrano, and J. F. Jimeno, "Drawing lessons from the boom of temporary jobs in Spain," The Economic Journal, vol. 112, no. 480, pp. F270-F295, 2002.

[5] K. Juselius and J. Ordonez, "The Balassa-Samuelson effect and the wage, price and unemployment dynamics in Spain," Discussion papers 05-29, Department of Economics, University of Copenhagen, Copenhagen, Denmark, 2005.

[6] D. Romero-Ávila and C. Usabiaga, “On the persistence of Spanish unemployment rates," Empirical Economics. In press.

[7] J. Ferreiro and F. Serrano, "The Spanish labour market: reforms and consequences," International Review of Applied Economics, vol. 15, no. 1, pp. 31-53, 2001.

[8] J. D. Hamilton, "A new approach to the economic analysis of nonstationary time series and the business cycle," Econometrica, vol. 57, no. 2, pp. 357-384, 1989.

[9] M. A. León-Ledesma and P. McAdam, "Unemployment, hysteresis and transition," Scottish Journal of Political Economy, vol. 51, no. 3, pp. 377-401, 2004.

[10] Z. Psaradakis and N. Spagnolo, "On the determination of the number of regimes in Markov-switching autoregressive models," Journal of Time Series Analysis, vol. 24, no. 2, pp. 237-252, 2003.

[11] G. Pisauro, "The effect of taxes on labour in efficiency wage models," Journal of Public Economics, vol. 46, no. 3, pp. 329-345, 1991.

[12] B. Lockwood and A. Manning, "Wage setting and the tax system theory and evidence for the United Kingdom," Journal of Public Economics, vol. 52, no. 1, pp. 1-29, 1993.

[13] S. Delipalla and P. Sanfey, "Commodity taxes, wage determination and profits," Journal of Public Economic Theory, vol. 3, no. 2, pp. 203-217, 2001. 
[14] J. R. Faria, "The effects of taxes on labour in a dynamic efficiency wage model," The Japanese Economic Review, vol. 55, no. 3, pp. 286-297, 2004.

[15] E. Phelps, Structural Slumps: The Modern Equilibrium Theory of Unemployment, Interest, and Assets, Harvard University Press, Cambridge, Mass, USA, 1994.

[16] S. Nickell and R. Layard, "Labour market institutions and economic performance," in Handbook of Labor Economics, Vol. 3, O. Ashenfelter and D. Card, Eds., pp. 3029-3084, North-Holland, Amsterdam, The Netherlands, 1999.

[17] F. Ostrup, Money and the Natural Rate of Unemployment, Cambridge University Press, Cambridge, UK, 2000.

[18] O. Blanchard and J. F. Jimeno, "Structural unemployment: Spain vs Portugal," The American Economic Review, vol. 85, no. 2, pp. 212-218, 1995.

[19] J. F. Jimeno, "Labor market reforms: lessons from Spain," in Beyond Transition: Development Perspectives and Dilemmas, M. Dabrowski, B. Slay, and J. Neneman, Eds., pp. 101-118, Ashgate, Hampshire, UK, 2004.

[20] G. Brunello and D. Sonedda, "Progressive taxation and wage setting when unions strategically interact," Oxford Economic Papers, vol. 59, no. 1, pp. 127-140, 2007.

[21] X. Raurich, H. Sala, and V. Sorolla, "Unemployment, growth, and fiscal policy: new insights on the hysteresis hypothesis," Macroeconomic Dynamics, vol. 10, no. 3, pp. 285-316, 2006.

[22] D. Ludwig, D. D. Jones, and C. S. Holling, "Qualitative analysis of insect outbreak systems: the spruce budworm and forest," The Journal of Animal Ecology, vol. 47, no. 1, pp. 315-332, 1978.

[23] R. Thom, Structural Stability and Morphogenesis, W. A. Benjamin, Reading, Mass, USA, 1976.

[24] E. C. Zeeman, “Catastrophe theory," Scientific American, vol. 234, no. 4, pp. 65-83, 1976.

[25] O. J. Blanchard and L. H. Summers, "Hysteresis and the European unemployment problem," in NBER Macroeconomics Annual, Vol. 1, pp. 15-90, MIT Press, Cambridge, Mass, USA, 1986.

[26] K. Røed, "Hysteresis in unemployment," Journal of Economic Surveys, vol. 11, no. 4, pp. 389-418, 1997.

[27] M. Camarero, J. L. Carrion-i-Silvestre, and C. Tamarit, "Testing for hysteresis in unemployment in OECD countries: new evidence using stationarity panel tests with breaks," Oxford Bulletin of Economics and Statistics, vol. 68, no. 2, pp. 167-182, 2006.

[28] A. Jaeger and M. Parkinson, "Some evidence on hysteresis in unemployment rates," European Economic Review, vol. 38, no. 2, pp. 329-342, 1994. 


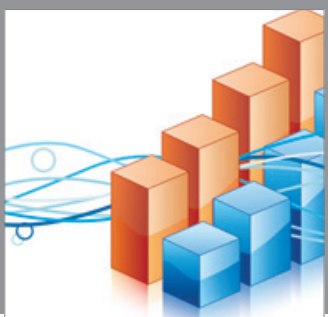

Advances in

Operations Research

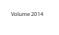

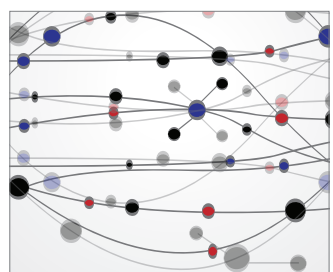

\section{The Scientific} World Journal
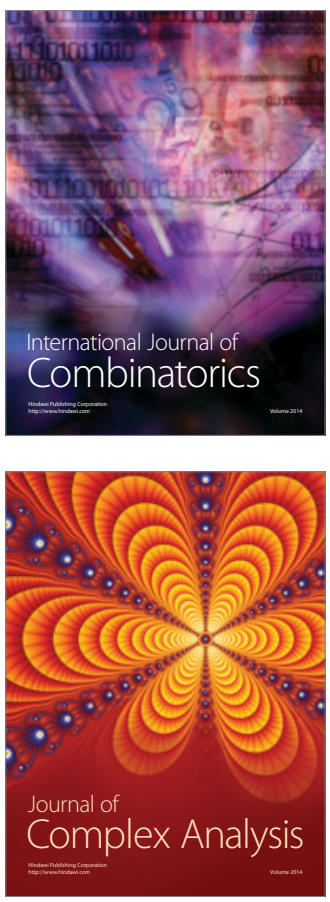

International Journal of

Mathematics and

Mathematical

Sciences
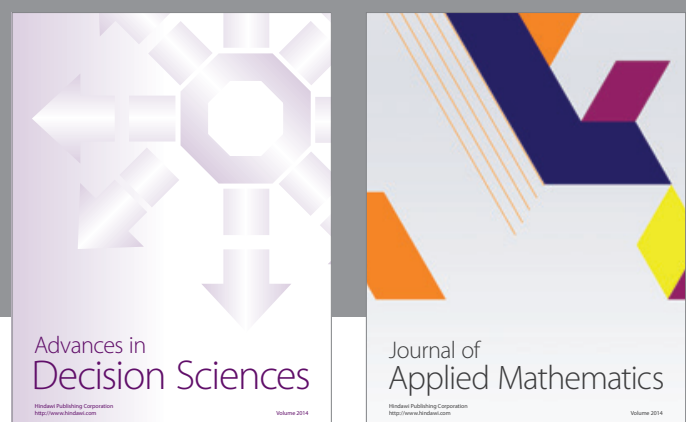

Journal of

Applied Mathematics
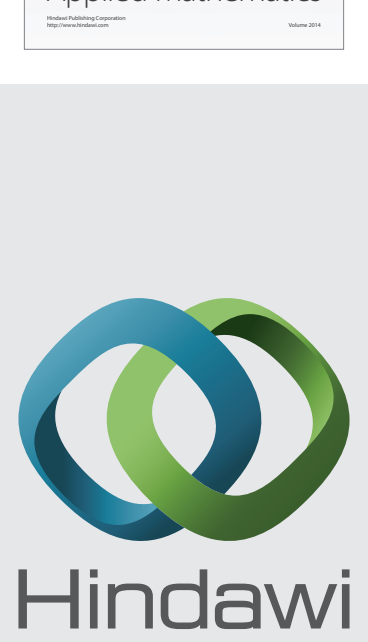

Submit your manuscripts at http://www.hindawi.com
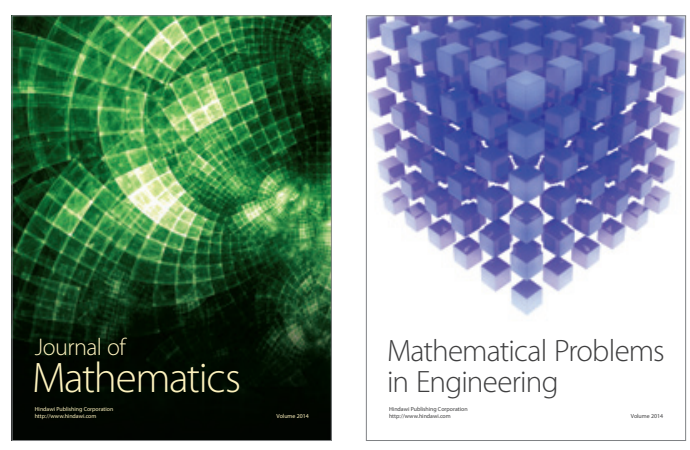

Mathematical Problems in Engineering
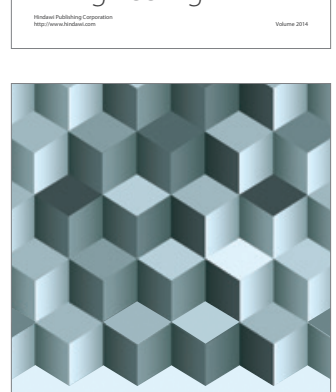

Journal of

Function Spaces
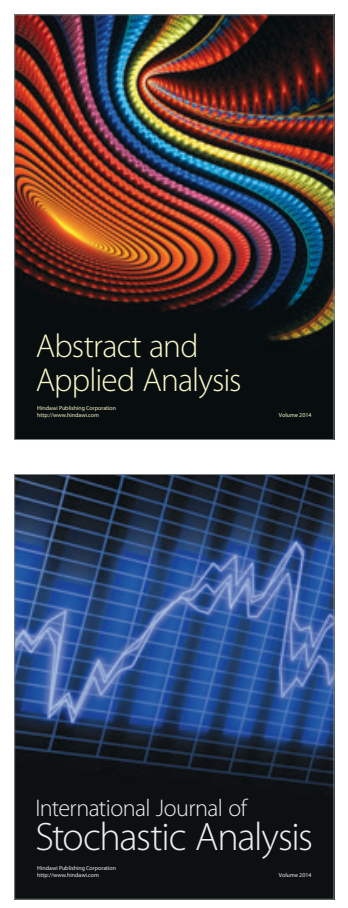

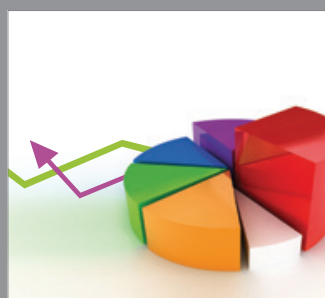

ournal of

Probability and Statistics

Promensencen
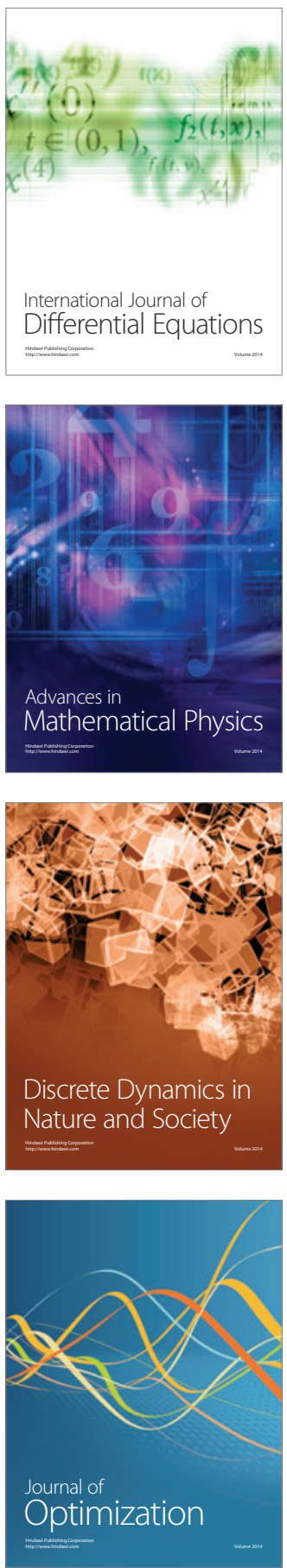